\title{
Validation of an Experimentally Derived Uncertainty Model
}

\author{
K. B. Lim* and D. E. Cox ${ }^{\dagger}$ \\ NASA Langley Research Center, Hampton, Virginia 23681 \\ G. J. Balas \\ University of Minnesota, Minneapolis, Minnesota 55455 \\ and \\ J.-N. Juang ${ }^{\S}$ \\ NASA Langley Research Center, Hampton, Virginia 23681
}

\begin{abstract}
The discrepancies between measurement data and an analytical nominal model for a large gap magnetic suspension testbed is accounted for by an uncertainty model. The results show that uncertainty bounds corresponding to a combination of additive and diagonal input multiplicative uncertainty can be obtained directly by calculating the smallest norm of the difference between the measured and nominal model response. Use of the identified uncertainty model allowed a strong correlation between design predictions and experimental results. In addition, robust controllers based on the experimentally derived uncertainty model show significant stability and performance improvements over controllers designed with assumed ad hoc uncertainty levels.
\end{abstract}

\section{Introduction}

$\mathbf{T}$ HE use of a linear, time-invariant (LTI) and finite dimensional model is still an approximation of a true plant in many engineering problems. The need for robust control is often due to the corruption of the measurement data by the secondary effects of measurement noise, external disturbances, nonlinearities, and possible time variations. Although, strictly speaking, robust control theory for nonlinear, time-varying systems should be applied under the aforementioned circumstances, it is currently not available. However, it has been shown that LTI-based robustness theory can handle a class of time-varying and/or nonlinear uncertainties or effects. ${ }^{1-4}$ The hope is that a small set of LTI plants will be sufficient to describe these secondary effects.

A method has been proposed recently ${ }^{5,6}$ for calculating the smallest norm of the difference between the raw systemidentification data and the predicted value from a given nominal model. In particular, this minimum norm is calculated in closed form (to within a linear matrix equation) and holds for a general nominal/uncertainty structure in linear fractional transformation (LFT) form. For use in this paper, a set of plants is said to be model validating with respect to given measured input/output data if the measured data can be reproduced from the set of plants. The method computes the smallest subset of plants among all model validating sets of plants about the nominal, hence the name, minimum norm model validation (MNMV).

This paper is an attempt to demonstrateand to validate the MNMV approach for methodically constructing uncertainty models for a real application. As part of a validation, the uncertainty weights identified are used in redesigning the controller and comparing the experimental closed-loop performance to controllers that assume ad hoc uncertainty levels. The feasibility of the method is investi-

\footnotetext{
Received Nov. 26, 1996; presented as Paper 97-0244 at the AIAA 35th Aerospace Sciences Meeting, Reno, NV, Jan. 6-9, 1997; revision received Sept. 30, 1997; accepted for publication Sept. 30, 1997. Copyright (C) 1997 by the American Institute of Aeronautics and Astronautics, Inc. No copyright is asserted in the United States under Title 17, U.S. Code. The U.S. Government has a royalty-free license to exercise all rights under the copyright claimed herein for Governmental purposes. All other rights are reserved by the copyright owner.

${ }^{*}$ Research Engineer, Guidance and Control Branch, Flight Dynamics and Control Division, MS 161. E-mail: k.b.lim@larc.nasa.gov.

${ }^{\dagger}$ Research Engineer, Guidance and Control Branch, Flight Dynamics and Control Division, MS 161. E-mail: d.e.cox@1arc.nasa.gov.

Associate Professor, Department of Aerospace Engineering and Mechanics, 107 Akerman Hall, 110 Union Street S.E. E-mail: balas@jette.aem. umn.edu. Member AIAA.

${ }^{\S}$ Principal Scientist, Structural Dynamics Branch, Structures Division, MS 230. E-mail: j.juang @larc.nasa.gov. Fellow AIAA.
}

gated by applying it to the large angle magnetic suspension test fixture (LAMSTF). ${ }^{7}$ The LAMSTF is an experimental testbed located at NASA Langley Research Center for precision pointing control studies in support of large gap magnetic suspension technology. The LAMSTF system is open loop unstable; hence system uncertainty identification (UID) is performed closed loop.

An analytical model as derived in Refs. 8-10 is used as the nominal model in this study although an identified model can be obtained and used as a nominal model from the same UID data. In principle, it should not matter which nominal model is used so long as the set of plants can be described without stretching the uncertainty size. Hence, any nominal model, provided it is not too far away from the true unknown system, could be used. For example, an observerbased system identification (ID) technique $e^{11-13}$ may be used to construct a single best nominal model, and the residual discrepancies between the raw system ID data and the nominal model are bounded by a structured uncertainty connection, assumed a priori. ${ }^{14,15}$ This would yield an uncertainty bound directly from empirical data for use in robust controller design and analysis.

The paper is organized as follows. Section II summarizes the method used in the determination of uncertainty models by the MNMV approach. In Sec. III, the LAMSTF system is described briefly, and the experimental configuration and UID parameters are described. This is followed by results from UID experiments. Section IV describes how a series of controllers are designed, tested, and then compared in terms of stability robustness and disturbance rejection performance. Conclusions are given in Sec. V.

\section{UID Algorithm}

The general form of the model structure is given in Fig. 1. Using a bounded, but unknown, structured uncertainty model, a set of plants are defined that validates the available input-outputdata, which contains the deviation or scatter about the nominal. The input-output relationship can be written as

$$
y=F_{u}(P, \Delta) u+H w^{\prime}
$$

where $y, u$, and $w^{\prime}$ denote the output, input, and output noise. The upper LFT is defined by

$$
F_{u}(P, \Delta)=P_{22}+P_{21} \Delta\left(I-P_{11} \Delta\right)^{-1} P_{12}
$$

where $P$ denotes the augmented plant. The important point is that if the nominal $P_{22}$ is known, the rest of the augmented plant can be constructed from a priori assumptions on the uncertainty structure. Note that $H$ is the filter model for the noise, and we let the uncertainty $\Delta$ belong to the set of structured uncertainty $\mathcal{D}$, i.e., $\Delta \in \mathcal{D}$ (Ref. 14). 


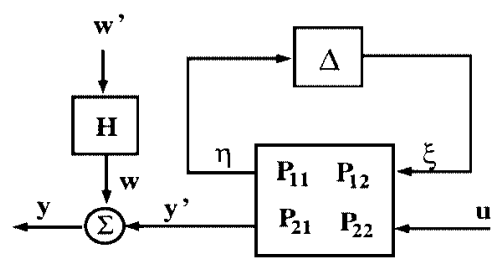

Fig. 1 A priori model structure of plant in standard form.

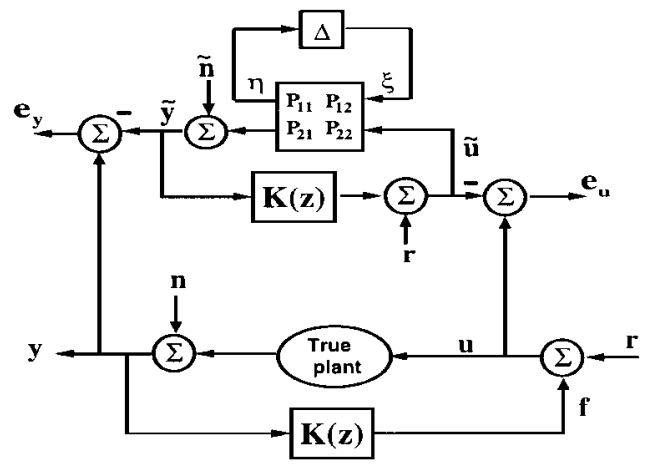

Fig. 2 Configuration for system UID.

Let the overall structured uncertainty be defined by the block diagonal matrices

$$
\Delta=\operatorname{diag}\left(\Delta^{1}, \ldots, \Delta^{\tau}\right), \quad \Delta^{j} \in C^{m_{j} \times n_{j}}
$$

and let the set of all block diagonal and stable, rational transfer function matrices be given by

$$
\mathcal{D}=\left\{\Delta(\cdot) \in R H_{\infty}: \Delta^{j}\left(s_{o}\right) \in C^{m_{j} \times n_{j}}, \forall s_{o} \in \bar{C}_{+}\right\}
$$

where $\tau$ and $\bar{C}_{+}$denote the number of uncertainty blocks and the closed right-halfplane, respectively. ${ }^{14}$ We considerthe class of problems where the uncertainty connections to the nominal and the plant inputs and outputs are given. In the next section, these deviations from a nominal model are used to develop unconservative frequency-dependert structured uncertainty models.

To account for the discrepancies between the available measured outputs and feedback signals and their estimates from a nominal model, a priori knowledge of possible sources of uncertainties in the system are used. Figure 2 shows how a nominal model $P_{22}$ and an uncertainty connection structure can be used to define the LFT parameterized set of plants with output noise. The direct connection between the structured uncertainties $\Delta$ to the output and input mismatch $e_{y}$ and $e_{u}$ is shown. These errors are defined by

$$
e:=\left\{\begin{array}{l}
e_{y} \\
e_{u}
\end{array}\right\}=\left\{\begin{array}{l}
y \\
u
\end{array}\right\}-\left\{\begin{array}{l}
\tilde{y} \\
\tilde{u}
\end{array}\right\}
$$

It is important to note that the preceding errors are the residuals that remain after a single best model fit. This error is usually discarded in standard system ID applications. However, this residual error is precisely the data used in generating uncertainty models. It is clear that this error is composed of errors due to model mismatch and errors due to filtered noise. Note that the error expression in the estimated output for closed-loop ID is the same as in the open loop. ${ }^{5}$

The algorithm used to identify uncertainty bounds is given in Ref. 5 for the open-loop case and in Ref. 6 for the closed-loop case. In both cases, the norm of the smallest structured uncertainty that validates the available ID data at each frequency is found, i.e., a minimum norm model validation.

It is assumed that the controller dynamics $K$ are known and that the plant inputs, $u \in R^{n_{u} \times 1}$, and outputs, $y \in R^{n_{y} \times 1}$, are measured while the external command, $r \in R^{n_{r} \times 1}$, is selected. The fictitious signals in Fig. 2 have dimensions $\eta \in C^{n_{\eta}}$ and $\xi \in C^{n_{\xi}}$, where

$$
n_{\eta}=\sum_{j=1}^{\tau} n_{j}, \quad n_{\xi}=\sum_{j=1}^{\tau} m_{j}
$$

The error in Eq. (5) is given by

$$
e=e_{o}-R_{21} \Delta\left(I-R_{11} \Delta\right)^{-1} M_{12}
$$

where

$$
\begin{gathered}
R_{11}=P_{11}+P_{12} K\left(I-P_{22} K\right)^{-1} P_{21}=F_{l}(P, K) \\
R_{12}=P_{12}\left(I-K P_{22}\right)^{-1}\left[\begin{array}{ll}
I & K
\end{array}\right] \\
R_{21}=\left[\begin{array}{l}
I \\
K
\end{array}\right]\left(I-P_{22} K\right)^{-1} P_{21} \\
R_{22}=\left[\begin{array}{cc}
\left(I-P_{22} K\right)^{-1} P_{22} & \left(I-P_{22} K\right)^{-1} \\
\left(I-K P_{22}\right)^{-1} & \left(I-K P_{22}\right)^{-1} K
\end{array}\right] \\
e_{o}=\left\{\begin{array}{l}
y \\
u
\end{array}\right\}-R_{22}\left\{\begin{array}{l}
r \\
\tilde{n}
\end{array}\right\} \\
M_{12}=R_{12}\left\{\begin{array}{l}
r \\
\tilde{n}
\end{array}\right\}
\end{gathered}
$$

Note that $e_{o}$ is the residual from nominal fit when $\Delta=0$. Define the preceding residuals at the discrete frequencies

$$
\Omega=\left(z_{1}, \ldots, z_{n_{\omega}}\right), \quad z_{i}=e^{j \omega_{i} T}
$$

by taking the discrete Fourier transform of both discrete time signals and systems.

Under conditions that ensure that a model-validating solution exists, a solution to the MNMV problem is summarized as follows. Let

$$
w=P_{21}^{+}\left(I-P_{22} K\right) e_{o}^{y}
$$

The singular value decomposition is given by

$$
P_{21}=U S V^{*}
$$

where $U \in C^{n_{y} \times n_{y}}$ and $V \in C^{n_{\xi} \times n_{\xi}}$ are Hermitian matrices, $S \in$ $R^{n_{y} \times n_{\xi}}$ is a full rank diagonal matrix and $*$ denotes conjugate transpose. The pseudoinverse is given by

$$
P_{21}^{+}=V S^{+} U^{*}
$$

A lower bound on the $j$ th component uncertainty at frequency $z_{i}$ is given by

$$
\bar{\sigma}\left(\Delta^{j}\right)=\left\|\Delta^{j}\right\|_{2} \geq \frac{\left\|y^{j}\right\|_{2}}{\left\|x^{j}\right\|_{2}}
$$

when $x^{j} \neq 0$ and

$$
x:=M_{12}+R_{11}(w+\phi), \quad y:=w+\phi
$$

which are partitioned

$$
x=\left\{\begin{array}{c}
x^{1} \\
\vdots \\
x^{\tau}
\end{array}\right\}, \quad y=\left\{\begin{array}{c}
y^{1} \\
\vdots \\
y^{\tau}
\end{array}\right\}
$$

in a conformal manner with respect to $\tau$ uncertainty blocks. Let $\mathcal{N}$ denote the null space of $P_{21}$ and $\phi \in \mathcal{N}$. Assuming that $n_{\xi} \geq n_{y}$, it has dimension $n_{\xi}-n_{y}$ and is spanned by the last $n_{\xi}-n_{y}$ columns of $V$. A component uncertainty with this lower bound has been shown to exist. Thus the minimum norm bound $\delta_{j}$ for each uncertainty block can be computed from Eq. (18). For robust control design, the minimum bounds can be overbound by a stable, realizable low-order transfer function for each uncertainty block. 


\section{UID of LAMSTF}

Detailed descriptions of the LAMSTF facility and the open-loop dynamic properties of the magnetic suspension system are given in Ref. 8. Earlier studies on system ID and control for LAMSTF include Refs. 9, 10, and 16. For LAMSTF, the model uncertainty is due to errors in the linearization about the equilibrium state, an inaccurate knowledge of the spatial distribution of the magnetic field, errors in the sensor system hardware, and errors at the plant input due to induced eddy currents. Collectively, these uncertainties lead to a multitude of uncertain parameters and inevitable unmodeled dynamics. For simplicity, we make a sweeping assumption of lumping all of the uncertainties into a combination of additive and diagonal input multiplicative uncertainties.

\section{A. Analytical Model}

An analytical model is derived in Refs. 8-10 and reviewed only briefly here. Figure 3 shows a schematic of the LAMSTF system. It basically consists of five electromagnets that actively suspend a small cylindrical permanent magnet. The cylinder is a rigid body and has five independent degrees of freedom, with motion in the roll axis being both unobserved and uncontrolled.Reference 9 gives a detailed derivation of both the linear and nonlinear models. The following provides a synopsisfor the linear model used in the control design.

By defining the state vector

$$
\xi=\left(\omega_{2}, \omega_{3}, \beta_{2}, \beta_{3}, v_{1}, v_{2}, v_{3}, x_{1}, x_{2}, x_{3}\right)^{T}
$$

the linearized perturbed motion about the equilibrium is given by

$$
\delta \dot{\xi}=\hat{A} \delta \xi+\hat{B} \delta \rho
$$

where the detailed expressions for $\hat{A}$ and $\hat{B}$ are given in Ref. 9. Note that these analyticalexpressionsdepend on many physical constants, including a series approximation of the magnetic field distribution. In fact, this field distribution and its frequency dependence due to eddy currents are believed to be a primary source of frequency dependent model errors. The variables $\omega_{i}, \beta_{i}, v_{i}$, and $x_{i}$ denotes $i$ th angular velocity of cylinder with respect to body frame, $i$ th Euler parameter relative to inertial frame, $i$ th translational velocity, and displacement of the centroid, respectively. Six optical sensors detect in-plane and out-of-plane motion and provide an overdetermined set of measurements for position in $x, y$, and $z$ and rotation in pitch and yaw. The 6 optical sensors and 5 control coils yield a fully controllable and observable 10-state system whose eigenvalues are shown in Table 1.

The system's dynamics are dominated by the unstable pitch and yaw modes. These modes, called compass needle modes, result from the magnetic field being 180 deg out of phase with the cylinder's

Table 1 Eigenvalues of 10-state analytic model

\begin{tabular}{lc}
\hline \hline Analytic eigenvalues & Degrees of freedom \\
\hline $0.00 \pm 0.95 i$ & $z$ axis \\
$0.00 \pm 7.97 i$ & $x$ axis, $\theta_{y}$ \\
\pm 9.77 & $y$ axis \\
\pm 57.80 & $\theta_{z}$ \\
\pm 58.78 & $\theta_{y}, x$ axis \\
\hline \hline
\end{tabular}

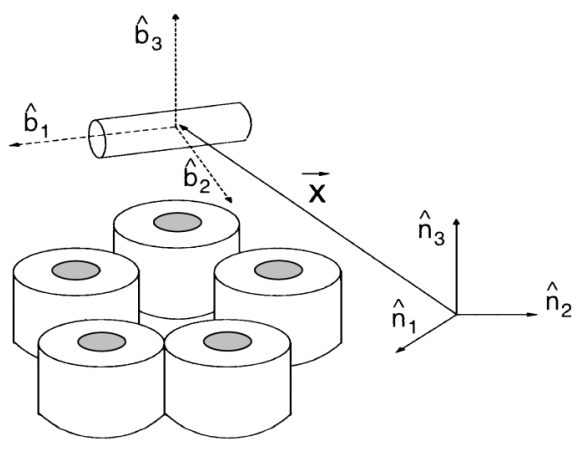

Fig. 3 LAMSTF configuration.
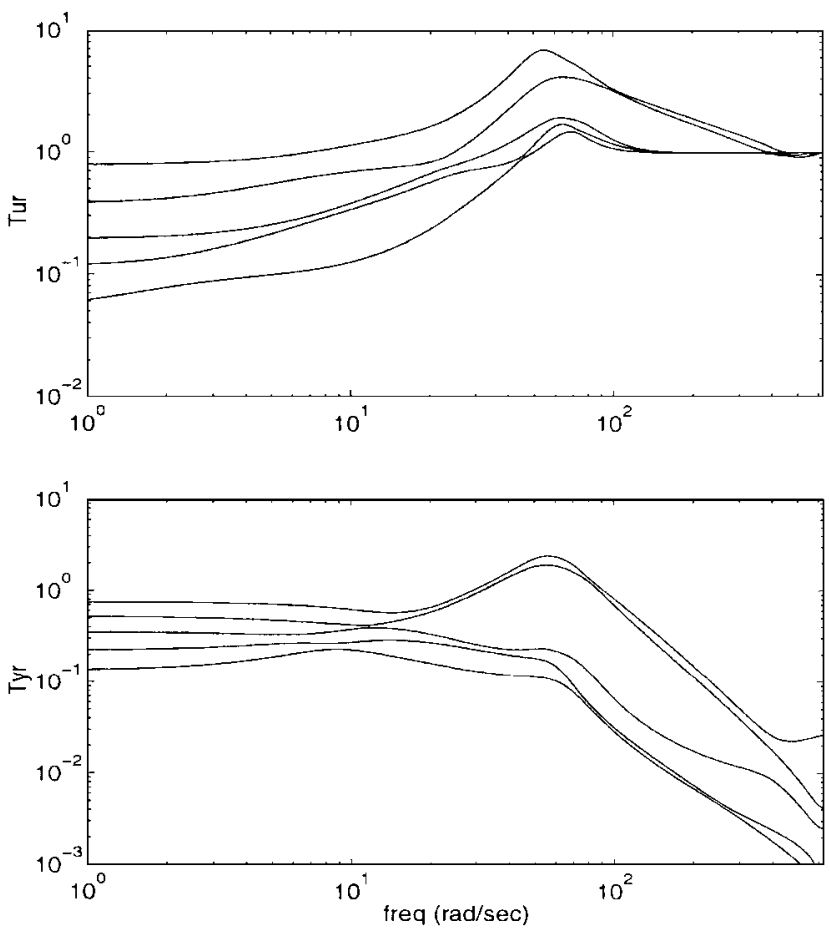

Fig. 4 Singular values of input sensitivity (top) and closed-loop transfer function across plant (bottom).

axial magnetization at the unstable equilibrium point. For a detailed discussion of the physical significance of all modes, the interested reader is referred to Ref. 8.

\section{B. UID Experiment}

The UID input test signal used consists of a frequency weighted, zero mean, white noise random signal for all five inputs $r$ (see Fig. 2). This was generated by filtering a white noise signal with standard deviation of $1 \mathrm{~A}$ through a fourth-order, low-pass Butterworth filter with a break frequency of $60 \mathrm{~Hz}$. A second random signal with a bandwidth of $2 \mathrm{~Hz}$ but with a standard deviation of $5 \mathrm{~A}$ is added to the $60-\mathrm{Hz}$ bandwidth signal. This second signal is used to alleviate the lack of power at low frequencies that may result in a poor model at these low frequencies. The total time of the excitation signal is about $40 \mathrm{~s}$ at a $200-\mathrm{Hz}$ sampling rate.

Because of the closed-loop coupling, the external UID signal introduced at the plant input is modified according to the unknown true input sensitivity transfer function matrix. Based on a nominal model, the top plot in Fig. 4 shows the input sensitivity matrix $T_{u r}=\left[I-F_{u}(P, \Delta) K\right]^{-1}$, whereas the bottom plot shows the closed-loop transfer function across the plant $T_{y r}=$ $\left[I-F_{u}(P, \Delta) K\right]^{-1} F_{u}(P, \Delta)$. Of course even an LFT model of the true plant denoted by $F_{u}(P, \Delta)$ is not known so that the best model before the test is used. Figure 4 shows that the UID input is attenuated both at the input and output of the plant at low frequencies. This is due to the inherent disturbance rejection property of the controller used in the experiment. Note that the maximum singular value at these low frequencies is an optimistic expectation of the actual response. The magnitudes of the input and outputs signals are largest at frequencies near $60 \mathrm{rad} / \mathrm{s}$. The output response to the UID signal is attenuated at higher frequencies. This means that an identified uncertainty may be limited in accuracy at both low and high frequencies.

\section{Uncertainty Model}

It is known that for this testbed there are several sources of uncertainties, which include errors in the linearization about the equilibrium state, inaccurate knowledge of the spatial distribution of the magnetic field, errors in the sensor system hardware, and errors at the plant input due to induced eddy currents. In particular, obtaining accurate analytical models of eddy currents using recently developed sophisticatedcomputer code is challenging especially with multiple eddy current circuits with complex geometries. ${ }^{17}$ 


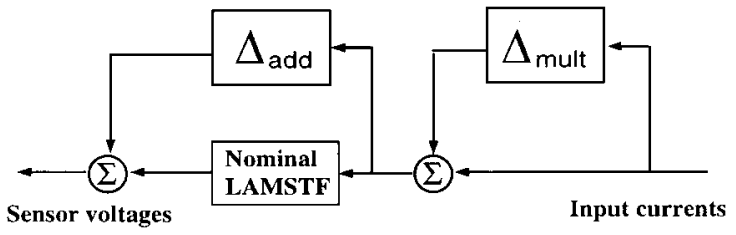

Fig. 5 Assumed uncertainty structure.

In this study, for simplicity we consider a combination of additive and structured input multiplicative uncertainty to describe the deviation of a nominal model. Another reason for choosing this uncertainty structure is to give a sufficient degree of uncertainty freedom to permit a model validating solution. Of course, in general, a methodical selection of the uncertainty connections or structure is still an open issue.

Figure 5 shows the assumed connectionsfor the uncertainties. The bounds on this structured uncertainty can then be experimentally determined by the minimum norm model validation method. The $P-\Delta$ connections are given by

$$
\begin{gathered}
\left\{\begin{array}{c}
\xi_{\text {add }} \\
\xi_{\text {mult }}
\end{array}\right\}=\left[\begin{array}{cc}
\Delta_{\text {add }} & 0 \\
0 & \Delta_{\text {mult }}
\end{array}\right]\left\{\begin{array}{c}
\eta_{\text {add }} \\
\eta_{\text {mult }}
\end{array}\right\} \\
\left\{\begin{array}{c}
\eta_{\text {add }} \\
\eta_{\text {mult }} \\
y
\end{array}\right\}=P\left\{\begin{array}{c}
\xi_{\text {add }} \\
\xi_{\text {mult }} \\
u
\end{array}\right\}
\end{gathered}
$$

where the structured multiplicative uncertainty is

$$
\Delta_{\text {mult }}=\operatorname{diag}\left(\delta_{1}, \ldots, \delta_{5}\right), \quad \delta_{i} \in C
$$

The corresponding augmented plant for the structured uncertainty is

$$
P=\left[\begin{array}{lll}
0 & I & I \\
0 & 0 & I \\
I & G & G
\end{array}\right]
$$

The preceding variables have the following dimensions: $\xi_{\text {add }} \in$ $C^{6 \times 1}, \xi_{\text {mult }} \in C^{5 \times 1}, \eta_{\text {add }} \in C^{5 \times 1}, \eta_{\text {mult }} \in C^{5 \times 1}, \Delta_{\text {add }} \in C^{6 \times 5}, u \in$ $R^{5 \times 1}$, and $y \in R^{6 \times 1}$.

The uncertainty bounds are calculated at discrete frequencies that are linearly spaced. A white noise signal having a spectral density of $10^{-6}$ is assumed for the presence of measurement noise. Because the number of uncertainty channels $\left(n_{\xi}=11\right)$ is greater than the number of outputs $\left(n_{y}=6\right), P_{21}$ is rectangular and its null space will be of minimum dimension 5 . This means that the uncertainty bounds can be further reduced if this null space freedom is utilized. However, this freedom is not considered in this study.

Figure 6 shows the calculated minimum norm additive uncertainty (dot) at discrete frequency points over the whole range of Nyquist frequency. The curvefit was performed interactively using the $\mu$-Too ${ }^{15}$ drawmag routine using a stable, rational, first-order transfer function (solid line). The maximum (dashed line) and minimum (dash-dot line) singular value response of the nominal plant are shown for comparison. It is seen that at frequencies below approximately $7 \mathrm{~Hz}$ the uncertainty is less than the minimum singular value response, indicating that the additive uncertainty levels are very small, whereas at higher frequencies the predicted additive uncertainty effect becomes more important. At frequencies beyond $60 \mathrm{~Hz}$, predicted uncertainty is unreliable because the ID input signal is bandlimited.

Figure 7 shows the calculated minimum norm multiplicative uncertainty (dot) and the corresponding second-order fit (solid line) for each input channel. The multiplicative uncertainty levels are generally larger at low frequencies and roll off with increasing frequencies.

Input channel number 1 shows largest uncertainty levels of up to about $90 \%$, but at frequencies near crossover (around $10 \mathrm{~Hz}$ ) the uncertainty levels of all input multiplicative uncertainties are about

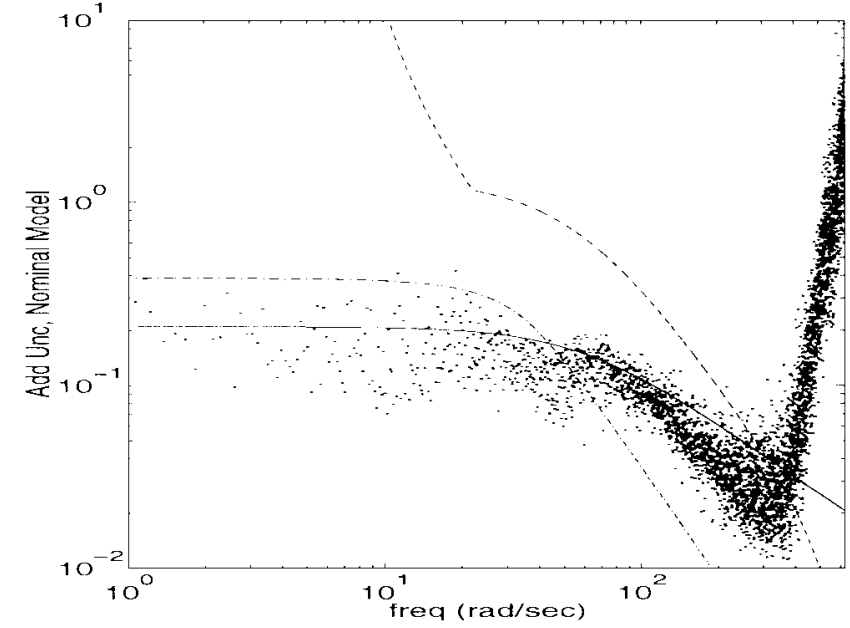

Fig. 6 Additive uncertainty: $\cdot$, predicted, and ——, uncertainty fit; and - - -, maximum, and - - -, minimum, singular value of nominal model.
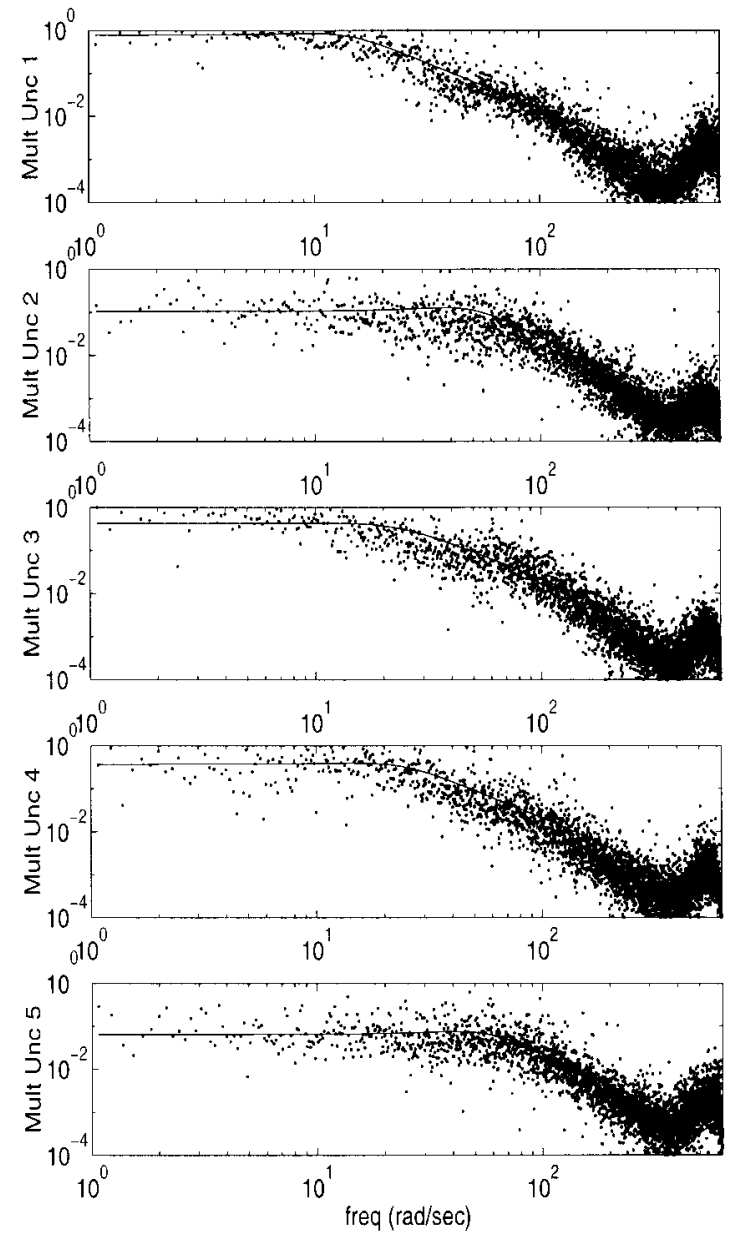

Fig. 7 Input multiplicative uncertainty: $\cdot$, predicted, and - , uncertainty fit.

$10 \%$ or less. However, each channel appears to have different break frequencies for the rolloff. For example, channel 1 having larger uncertainty levels at low frequencies than channel 2 rolls off at about $2 \mathrm{~Hz}$ as compared with about $10 \mathrm{~Hz}$ for channel 2 . Note also that these experimentally based uncertainty predictions are very different when compared with assumed constant uncertainty levels. The consequence of this difference is evident from experimental results.

\section{Performance Validation}

The main objective of the control design is to stabilize the highly unstable open-loop equilibrium configuration. Subject to closedloop stability, reasonable disturbance rejection performance across 
the plant is desired. Of course, the aforementioned objectives are complicated by the presence of model error inaccuracies, which are painfully evident during experiments. Hence, robust disturbance rejection is sought, which requires a definition of the uncertainty set for which the performance is guaranteed. Uncertainty in the uncertainty model itself is a real dilemma that motivates this study, which is a continuation of an earlier study. ${ }^{10}$ Of course, now we have the benefit of a new tool ${ }^{5,6}$ to more realistically capture model uncertainty.

\section{A. Controller Design}

Figure 8 shows the interconnection used in the design of $H_{\infty}$ and $\mu$ controllers. ${ }^{14}$ The robust performance (RP) is defined by the principal gains such that

$$
\bar{\sigma}\left\{W_{y}\left[I-F_{u}(P, \Delta)\right]^{-1} F_{u}(P, \Delta)\right\} \leq 1, \quad \forall \omega \in[0, \infty)
$$

where the set of plants defined by the uncertainty,

$$
\begin{array}{cc}
\Delta=\operatorname{blk}-\operatorname{diag}\left(\Delta_{\text {add }}, \Delta_{\text {mult }}\right) \\
\Delta_{\text {add }}=\hat{\Delta}_{\text {add }} W_{\text {add }}, \quad & \bar{\sigma}\left(\hat{\Delta}_{\text {add }}\right) \leq 1 \\
\Delta_{\text {mult }}=\hat{\Delta}_{\text {mult }} W_{\text {mult }}, & \bar{\sigma}\left(\hat{\Delta}_{\text {mult }}\right) \leq 1
\end{array}
$$

and the input multiplicative uncertainty has the diagonal form

$$
\begin{gathered}
\hat{\Delta}_{\text {mult }}=\operatorname{diag}\left(\hat{\delta}_{1}, \ldots, \hat{\delta}_{5}\right) \\
W_{\text {mult }}=\operatorname{diag}\left(w_{m 1}, \ldots, w_{m 5}\right)
\end{gathered}
$$

The additive uncertainties are fitted with the following first-order stable $s$-domain transfer function

$$
W_{\text {add }}=3.7708 \times 10^{-3} \frac{(s+3380.7)}{(s+60.615)} I_{5 \times 5}
$$

The multiplicativeuncertaintiesare fitted with the following secondorder stable transfer functions of the form

$$
w_{m_{i}}=k_{i} \frac{\left(s+Z_{i}\right)\left(s+Z_{i}^{*}\right)}{\left(s+P_{i}\right)\left(s+P_{i}^{*}\right)}, \quad i=1, \ldots, 5
$$

and the poles, zeros, and gains of the transfer functions are given in Table 2. The uncertainty weights for both the additive and multiplicative cases are subsequently discretized using the Tustin approximation of a continuous filter. The output performance weight $W_{y}$ is chosen to be a constant diagonal matrix and equal for all six output channels

To validate the experimentally derived uncertainty model, four sets of $\mu$ controllers are considered. Table 3 shows the four sets of

Table 2 Parameters for multiplicative uncertainty fit

\begin{tabular}{lccc}
\hline \hline Input & $P_{i}$ & $Z_{i}$ & $k_{i}$ \\
\hline 1 & $-7.915 \pm 12.26 i$ & $-19.80 \pm 381.5 i$ & $1.127 e-03$ \\
2 & $-22.57 \pm 44.16 i$ & $-14.92 \pm 430.2 i$ & $1.377 e-03$ \\
3 & $-14.89 \pm 17.74 i$ & $-15.78 \pm 420.5 i$ & $1.294 e-03$ \\
4 & $-13.92 \pm 19.96 i$ & $-14.01 \pm 418.3 i$ & $1.243 e-03$ \\
5 & $-27.32 \pm 49.56 i$ & $-30.91 \pm 427.1 i$ & $1.115 e-03$ \\
\hline \hline
\end{tabular}

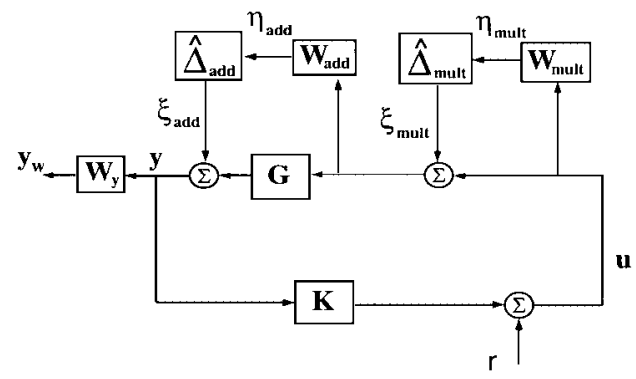

Fig. 8 Interconnection for robust controller design.
Table $3 \mu$ control laws tested

\begin{tabular}{lcccccc}
\hline \hline Controller & $W_{\text {unc }}$ & $W_{y}$ & $\mu_{\text {des }}$ & $\mu_{\mathrm{ID}}$ & $\|y\|_{2} /\|r\|_{2}{ }^{\mathrm{a}}$ & $\bar{\sigma}\left(T_{y r}\right)$ \\
\hline$C_{01}$ & 0.001 & 1 & 0.17 & 5.44 & $\mathrm{U}$ & $\overline{2.69}$ \\
$C_{02}$ & 0.1 & 1 & 1.89 & 2.89 & 0.29 & - \\
$C_{03}$ & 0.2 & 1 & 3.22 & 3.18 & $\mathrm{MU}$ & - \\
$C_{04}$ & 0.3 & 1 & 4.57 & 3.48 & $\mathrm{MU}$ & $\overline{1.40}$ \\
$C_{11}$ & IDed & 1 & 1.56 & 1.56 & 0.19 & - \\
$C_{022}$ & 0.1 & 0.1 & 1.41 & 5.63 & $\mathrm{U}$ & - \\
$C_{023}$ & 0.1 & 0.01 & 1.25 & 22.5 & $\mathrm{U}$ & $\overline{5.67}$ \\
$C_{12}$ & IDed & 0.1 & 1.29 & 5.85 & 0.42 & 26.4 \\
$C_{13}$ & IDed & 0.01 & 1.23 & 28.5 & 0.84 & \\
\hline \hline
\end{tabular}

${ }^{\mathrm{a}} \mathrm{U}=$ unstable and $\mathrm{MU}=$ marginally unstable.

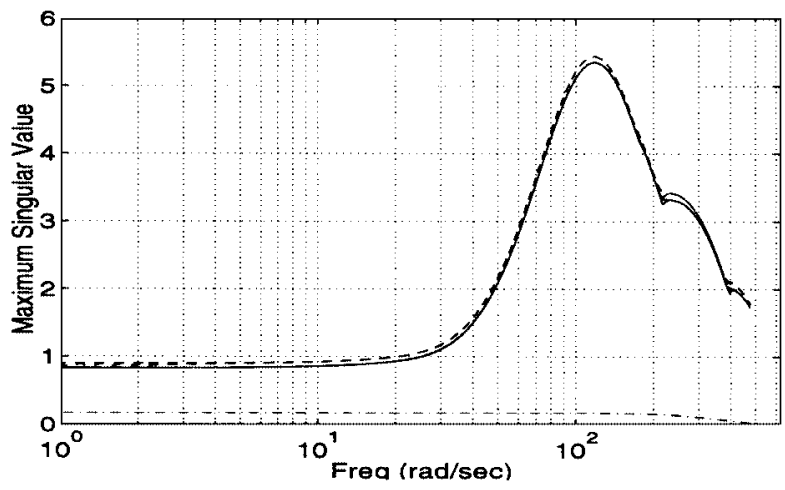

Fig. 9 Controller $C_{01}$ : predicted —-, RS; - --, NP; and ---, RP.

controllers that were designed and tested. In all cases, the analytical model is used as the nominal model. The first two sets of controllers are based on unity output weights, whereas the next two sets assume much smaller output weights of 0.1 and 0.01 .

The first set of control designs, $C_{01}, C_{02}, C_{03}$, and $C_{04}$, is based on assumed constant uncertainties at four different levels, $0.001,0.1$, 0.2 , and 0.3 , respectively. The second control design $C_{11}$ is based on an identified uncertainty model from Eqs. (33) and (34) and uses the same unity output weight as the first set. A third set of controllers, $C_{022}$ and $C_{023}$, are based on the assumed uncertainty level of 0.1 , which was used to design controller $C_{02}$, but with smaller output weights of 0.1 and 0.01 , respectively. The fourth and last set of controllers, $C_{12}$ and $C_{13}$, uses the scaled down output weights from the third set but with the identified uncertainty weights.

\section{B. Performance Comparison}

In this subsection, we evaluate the validity of the identified uncertainty model by comparing predicted robust stability (RS) and RP to actual experimental results.

\section{Stability Robustness}

In the first set of control designs, only controller $C_{02}$, which assumes uncertainty level of 0.1 , was stable. Controller $C_{01}$ was violently unstable, whereas controllers $C_{03}$ and $C_{04}$ were marginally unstable; i.e., it built up oscillations to eventually go out of range of the sensors. In contrast, a single control design, $C_{11}$, which is based on the identified uncertainty model, was stable and gave good performance without trial and error.

Based on the set of plants defined by the nominal and identified uncertainty model and the unity output weight, the predicted RS, nominal performance (NP), and RP were calculated for all nine controllers, resulting in Figs. 9-17. By comparing the predicted RS (solid line), the only four controllers that were actually stable$C_{11}, C_{12}, C_{13}$, and $C_{02}$ - have the four best predicted RS levels with respect to the identified uncertainty.

The two marginally unstable controllers, $C_{03}$ and $C_{04}$, have slightly worse predicted RS levels than the least stable controller, $C_{02}$, among the four stabilizing controllers. This suggests that the true system uncertainty level, lies in the narrow margin between $C_{03}$ (or $C_{04}$ ) and $C_{02}$.

The most violently unstable controller, $C_{01}$, corresponds to the worst RS prediction as shown in solid line in Fig. 9. This controller 


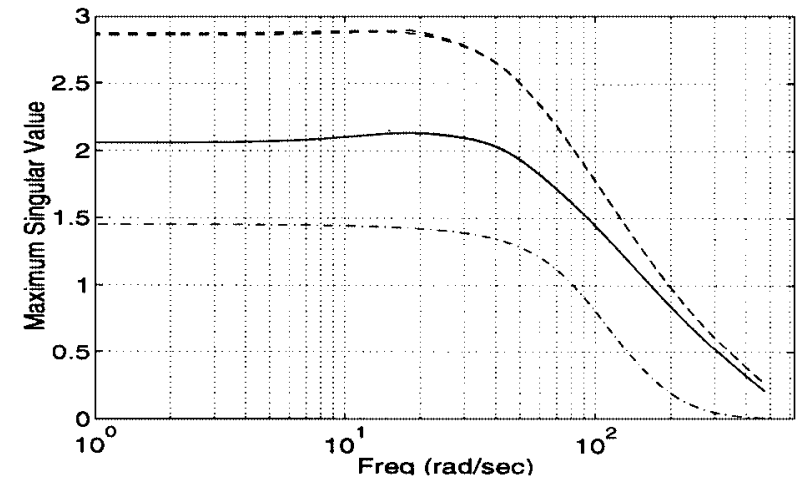

Fig. 10 Controller $C_{02}$ : predicted - , RS; - - -, NP; and ---, RP.

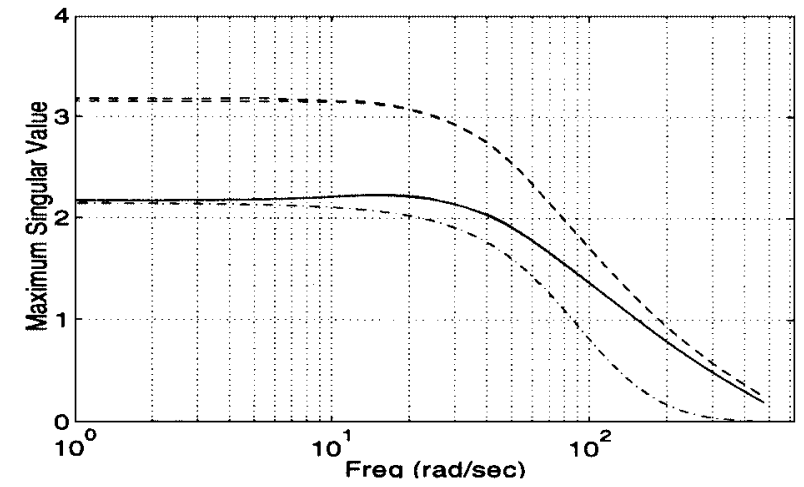

Fig. 11 Controller $C_{03}$ : predicted $\longrightarrow$, RS; ---, NP; and ---, RP.

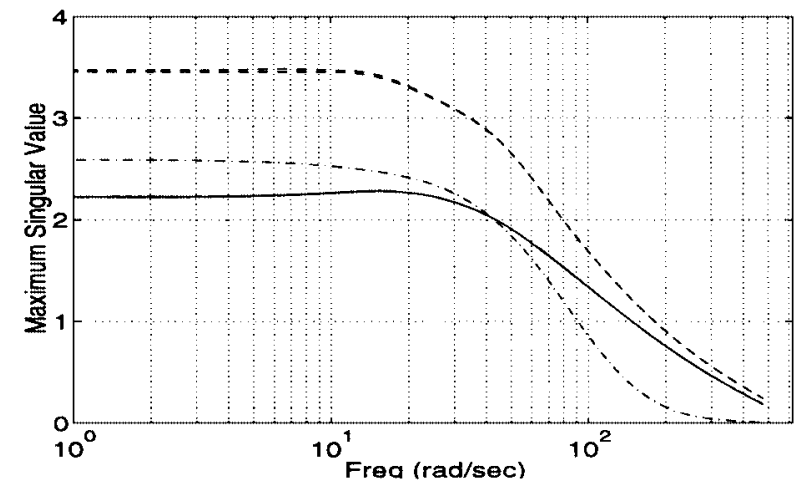

Fig. 12 Controller $C_{04}$ : predicted $\longrightarrow$, RS; - - -, NP; and ---, RP.

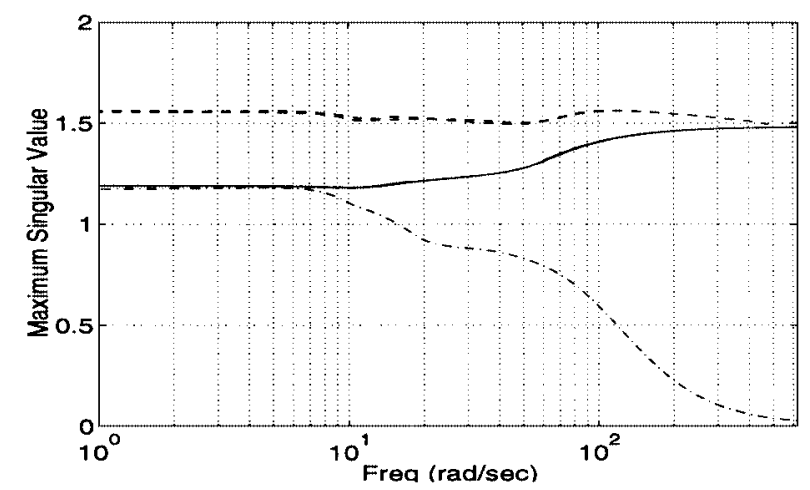

Fig. 13 Controller $C_{11}$ : predicted $\longrightarrow$, RS; - --, NP; and---, RP.

design practically ignores RS by assuming $W_{\text {unc }}=0.001 \times I_{10 \times 10}$. Note that because stability was attained in the four controllers in spite of a violation of RS condition (of less than unity) by a factor of up to 2 at lower frequencies, the identified uncertainty model is slightly conservative and the RS condition is only a sufficient condition for true-stability. However, a larger violation of RS condition as in controllers $C_{022}, C_{023}$, and $C_{01}$ (see Figs. 14, 15, and 9, respectively) results in an unstable closed-loop system.

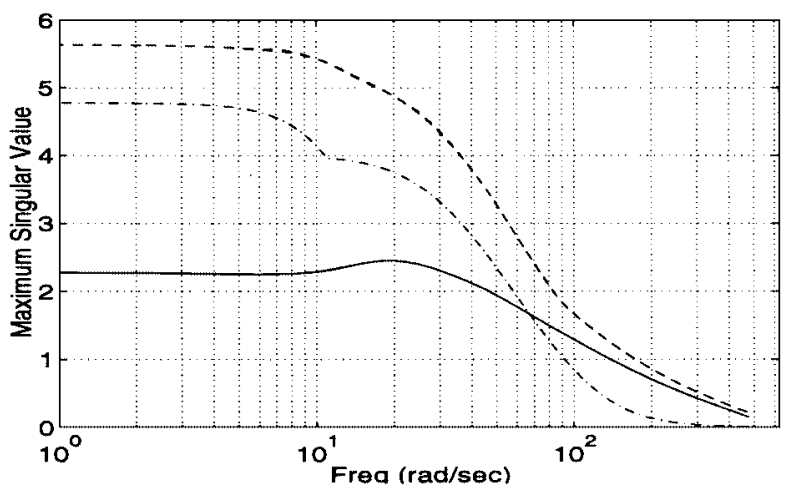

Fig. 14 Controller $C_{022}$ : predicted - , RS; ---, NP; and ---, RP.

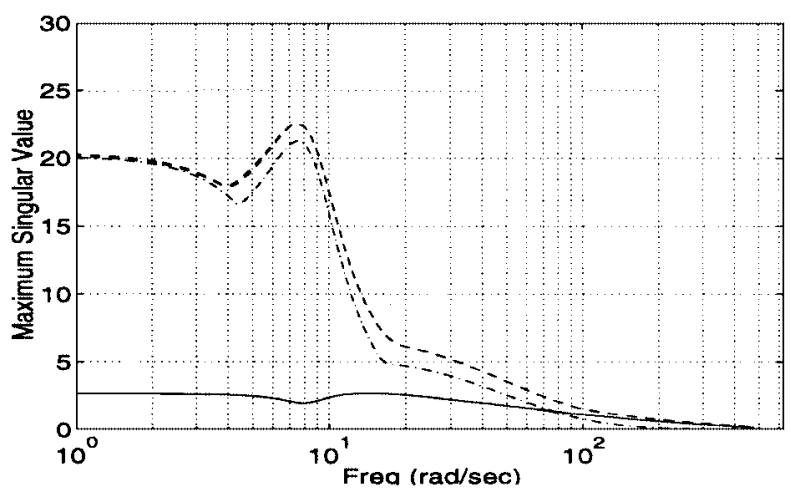

Fig. 15 Controller $C_{023}$ : predicted,- RS; ---, NP; and ---, RP.

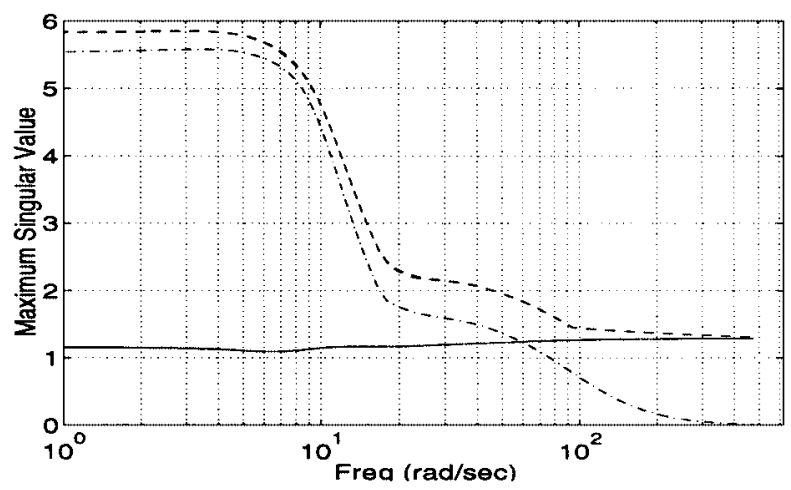

Fig. 16 Controller $C_{12}$ : predicted,- RS; - - -, NP; and---, RP.

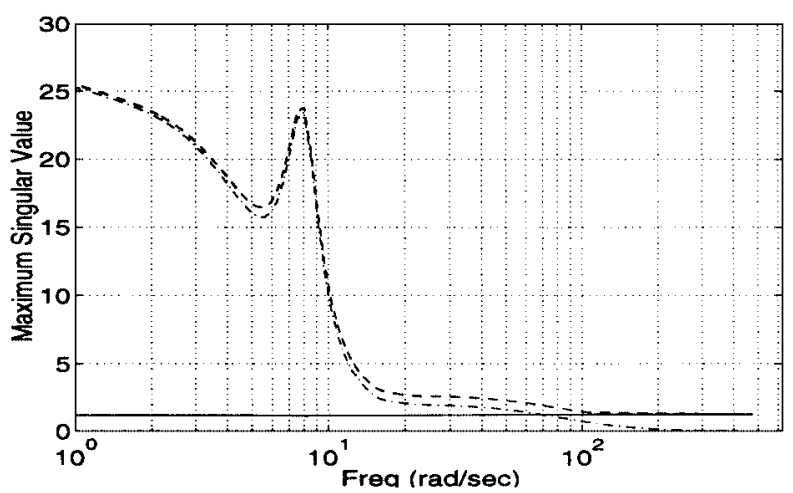

Fig. 17 Controller $C_{13}$ : predicted - , RS; - - -, NP; and---, RP.

Motivatedby the actual stability of controller $C_{02}$, controllers $C_{022}$ and $C_{023}$ were designed with reduced output weights, which resulted in an improvement in the predicted RP, namely, $\mu_{\mathrm{des}}$. If the assumed uncertainty of 0.1 is an accurate model, then reducing the output weight should lead to improved RS. Instead, controllers $C_{022}$ and $C_{023}$ were unstable when implemented. This instability is, however, consistent with the predicted degradation of RS as compared with $C_{02}$ when evaluated with the identified uncertainty model as shown 
in Figs. 14 and 15. In stark contrast, controllers $C_{12}$ and $C_{13}$ with reduced output weight were stable, although the disturbance rejection performance is poor as intended. This low performance but RS for controllers $C_{12}$ and $C_{13}$ can be seen from Figs. 16 and 17. Namely, the predicted poor RP is due to poor disturbance rejection at low frequency whereas a good RS level is maintained. This means that the identified uncertainty model used in controllers $C_{11}, C_{12}$, and $C_{13}$ displays a property of an accurate uncertainty model; namely, the RS condition should not depend on the choice of output weight.

\section{Robust Performance}

In Table $3, \mu_{\text {des }}$ denotes the designed $\mu$ with respect to the particular uncertainty model (assumed or identified) and output weights. The value $\mu_{\text {ID }}$ denotes the RP $\mu$ based on the identified uncertainty model and the fixed unity output weight. Thus, the differences between $\mu_{\text {des }}$ and $\mu_{\mathrm{ID}}$ values are solely due to the difference in both the uncertainty model assumed and the output weight. Therefore, $\mu_{\text {des }}$ equals $\mu_{\mathrm{ID}}$ only for $C_{11}$. In Table $3, \mu_{\mathrm{des}}, \mu_{\mathrm{ID}}$, and $\bar{\sigma}\left(T_{y r}\right)$ denote their respective maximum values over frequency.

Recall that a predicted RP is meaningful only with respect to the performance definition and the set of plants defined by the nominal and an uncertainty model. Clearly, if the set of plants assumed is in question, so is the reliability of the predicted RP. For instance, $\mu_{\text {des }}$ for controller $C_{01}$ is the smallest at 0.17 (see Table 3 ), but when implemented, the closed-loop system for this controller resulted in the most unstable system among the nine controllers tested. On the other hand, the best RP predicted by $\mu_{\mathrm{ID}}$ is controller $C_{11}$ at 1.56 . When tested, this controller actually gave the best performance out of the nine controllers.

To experimentally validate the RP predictions, a system ID experiment was conducted on the disturbance to output path of the closed-loop system. Identifying the system in this path allows the calculation of the directionwise worst-case response, i.e., the maximum singular value over all frequencies. A wideband uncorrelated disturbance input was added to each of the control coils, and measurements were recorded from the system's sensors. These data were used with the OKID algorithm ${ }^{11}$ to generate state-space models of the closed-loop system's disturbancepath, denoted as $T_{y r}$. The maximum singular values were then calculated to obtain the identified worst-case response subject to system ID limitations. These maximum singular values were directly compared with the $\mu_{\mathrm{ID}}$ bounds for RP. Figures 18-21 show these comparisons for all four stabilizing controllers over all frequencies.

For the controller pair $C_{02}$ and $C_{11}$, which have good RP, the experimental system had a worst-case performance just below the predicted $\mu$ bound, as shown in Figs. 18 and 19. This supports the earlier observations involving RS; namely, the identified uncertainty model was found to be slightly conservative so that the predicted $\mu$ is expected to be above the identified experimental worst-case response. The consistency between the identified experimental worstcase response and the predicted worst-case performance (based on the identified uncertainty model) makes a strong case for the accuracy of the uncertainty model. The second pair of controllers, $C_{12}$ and $C_{13}$, were dominated by RS constraints (see Figs. 16 and 17) and had poor predicted NP and RP. However, even in these cases there

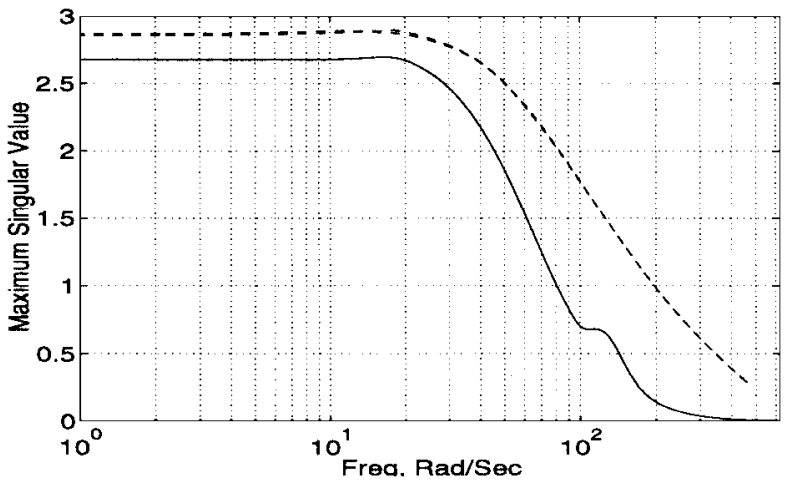

Fig. 18 Identified worst-case response (for controller $C_{02}$.

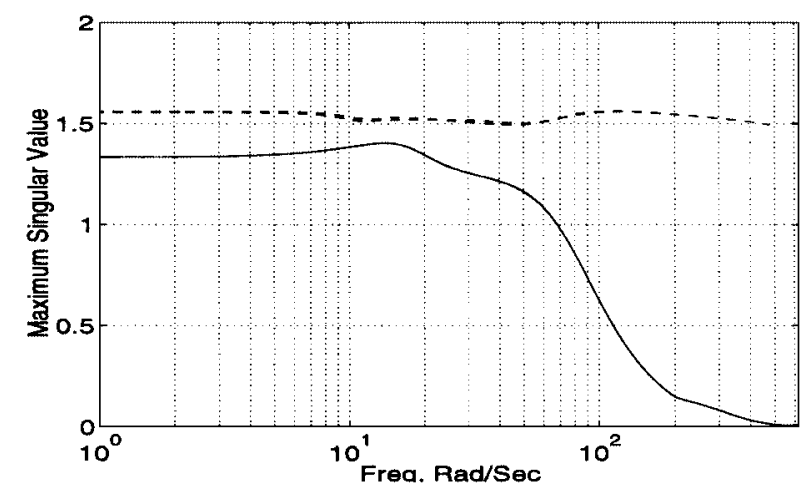

Fig. 19 Identified worst-case response (- ) and predicted RP (---) for controller $C_{11}$.

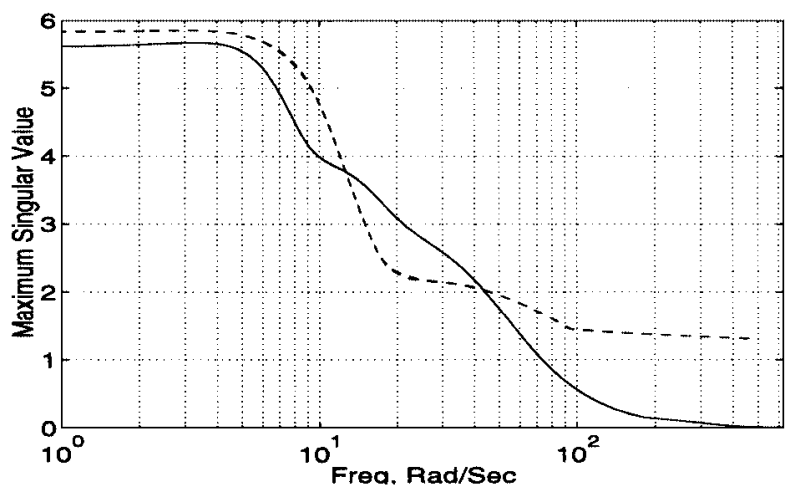

Fig. 20 Identified worst-case response (- $)$ and predicted RP (---) for controller $C_{12}$.

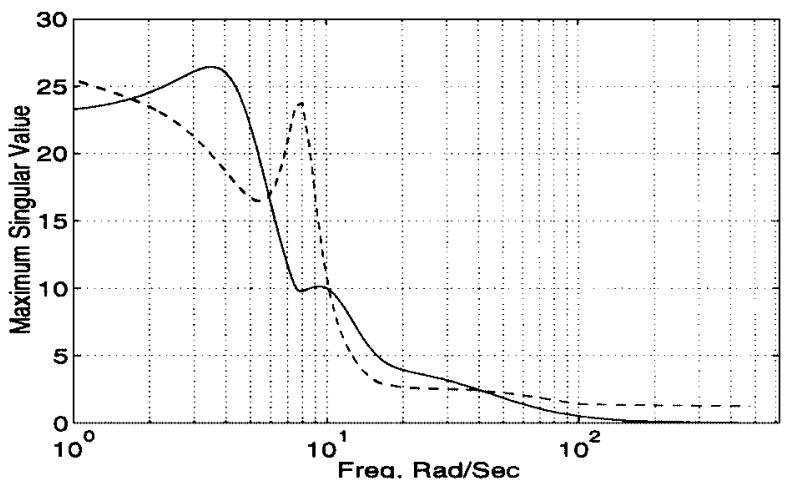

Fig. 21 Identified worst-case response (- - ) and predicted RP (---) for controller $C_{13}$.

is still a remarkable correlation between the predicted worst-case performance and the maximum singular values of the identified disturbance path, as shown in Figs. 20 and 21.

The last two columns in Table 3 show the ratio of signal 2 norms between the wideband white noise disturbance, the outputs $\|y\|_{2} /\|r\|_{2}$, and the maximum singular value of an identified model $T_{y r}$. From Table 3 it can be seen that for all four stabilizing controllers the following relation is satisfied:

$$
\frac{\|y\|_{2}}{\|r\|_{2}} \ll \bar{\sigma}\left(T_{y r}\right) \approx \mu_{\mathrm{ID}}
$$

The predicted and measured worst-case response matches approximately while they clearly bound a particular ratio of signal 2 norms.

\section{Conclusions}

This study demonstrated the use of a recently proposed algorithm for determining frequency-dependentbounds of uncertainty models directly from system identification test data. Overall, there was a strong correlation between actual and predicted RS and 
performance. Because this large gap magnetic suspension testbed is highly unstable in open loop and is sensitive to model errors, these results represent a significant experimental demonstration of the identified uncertainty model and subsequent robust controller performance. Hence, we conclude that the results validate the identified uncertainty model.

Robust control design based on the identified uncertainty model produced significantly better performance in terms of stability and RP. Use of the identified uncertainty model also significantly improved predictability. This reduces the need to tweak around both performance and uncertainty weights in a robust control design and subsequent application for real systems to obtain satisfactory performance.

The RS and performance results indicate that the identified uncertainty was not overly conservative. However, the null freedom available for the sufficiently parameterized uncertainty freedoms was not used to reduce the minimum norm uncertainty bound. Although the experimental results given in this study are based on a particular structure of uncertainty, namely, a combined additive and diagonal input multiplicative uncertainties, the system uncertainty identification methodology applies to the general linear fractional transformation framework.

\section{References}

${ }^{1}$ Safonov, M. G., Stability and Robustness of Multivariable Feedback Systems, MIT Press, Cambridge, MA, 1980, Chap. 2.

${ }^{2}$ Zames, G., "On the Input-Output Stability of Time-Varying Nonlinear Feedback Systems-Part I," IEEE Transactions on Automatic Control, Vol. AC-11, No. 2, 1966, pp. 228-238.

${ }^{3}$ Waszak, M. R., "A Methodology for Computing Uncertainty Bounds of Multivariable Systems Based on Sector Stability Theory Concepts," NASA TP 3166, April 1992.

${ }^{4}$ Poola, K., and Tikku, A., "Robust Performance Against Time-Varying Structured Perturbations," IEEE Transactions on Automatic Control, Vol. 40, No. 9, 1995, pp. 1589-1602.

${ }^{5}$ Lim, K. B., Balas, G. J., and Anthony, T. C., "Minimum-Norm Model
Validating Identification for Robust Control,” AIAA Paper 96-3717, July 1996.

${ }^{6} \mathrm{Lim}, \mathrm{K}$. B., "Closed Form Solution for Minimum Norm Model Validating Uncertainty," American Control Conf., AIAAF-97050, Albuquerque, NM, June 1997.

${ }^{7}$ Groom, N. J., "Description of the Large Gap Magnetic Suspension System (LGMSS) Ground-Based Experiment," Technology 2000, NASA CP3109, Vol. 2, 1991, pp. 365-377.

${ }^{8}$ Groom, N. J., and Britcher, C. P., "Open-Loop Characteristics of Magnetic Suspension Systems Using Electromagnets Mounted in a Planar Array," NASA TP 3229, Nov. 1992.

${ }^{9}$ Lim, K. B., and Cox, D. E., "Robust Tracking Control of a Magnetically Suspended Rigid Body," 2nd International Symposium on Magnetic Suspension Technology, NASA CP 3247, Aug. 1993.

${ }^{10}$ Lim, K. B., and Cox, D. E., "Experimental Robust Control Studies on an Unstable Magnetic Suspension System," American Control Conf., Baltimore, MD, June 1994, pp. 3198-3203.

${ }^{11}$ Juang, J.-N., Phan, M., Horta, L. G., and Longman, R. W., "Identification of Observer/Kalman Filter Markov Parameters: Theory and Experiments," Journal of Guidance, Control, and Dynamics, Vol. 16, No. 2, 1993, pp. 320-329.

${ }^{12}$ Juang, J.-N., and Phan, M., "Identification of System, Observer, and Controller from Closed-Loop Experimental Data," Journal of Guidance, Control, and Dynamics, Vol. 17, No. 1, 1994,pp. 91-96.

${ }^{13}$ Juang, J.-N., Applied System Identification, Prentice-Hall, Englewood Cliffs, NJ, 1994, Chap. 8.

${ }^{14}$ Stein, G., and Doyle, J. C., "Beyond Singular Values and Loop Shapes,' Journal of Guidance, Control, and Dynamics, Vol. 14, No. 1, 1991, pp. 5-16.

${ }^{15}$ Balas, G. J., Doyle, J. C., Glover, K., Packard, A., and Smith, R., $\mu-$ Analysis and Synthesis Toolbox, User's Guide, The MathWorks, Inc., Natick, MA, 1995.

${ }^{16}$ Groom, N. J., and Schaffner, P. R., "An LQR Controller Design Approach for a Large Gap Magnetic Suspension System (LGMSS)," NASA TM-101606, 1990.

${ }^{17}$ Britcher, C. P., and Groom, N. J., "Eddy Current Computations Applied to Magnetic Suspensions and Magnetic Bearings," Proceedings of MAG '95: Magnetic Bearings, Magnetic Drives and Dry Gas Seals Conference and Exhibition (Alexandria, VA), Technomic, Lancaster, PA, 1995, pp. 333342 . 